\title{
PAPER \\ Test-Retest Reliability and Criterion-Related Validity of the Implicit Association Test for Measuring Shyness
}

\author{
Tsutomu FUJII ${ }^{\dagger, \dagger \mathrm{a})}$, Member, Takafumi SAWAUMI ${ }^{\dagger \dagger, \uparrow \dagger}$, and Atsushi AIKAWA ${ }^{\dagger \dagger \dagger, \uparrow \dagger}$, Nonmembers
}

\begin{abstract}
SUMMARY This study investigated the test-retest reliability and the criterion-related validity of the Implicit Association Test (IAT [1]) that was developed for measuring shyness among Japanese people. The IAT has been used to measure implicit stereotypes, as well as self-concepts, such as implicit shyness and implicit self-esteem. We administered the shyness IAT and the self-esteem IAT to participants $(N=59)$ on two occasions over a one-week interval (Time 1 and Time 2 ) and examined the test-retest reliability by correlating shyness IATs between the two time points. We also assessed the criterion-related validity by calculating the correlation between implicit shyness and implicit self-esteem. The results indicated a sufficient positive correlation coefficient between the scores of implicit shyness over the one-week interval $(r=.67, p<.01)$. Moreover, a strong negative correlation coefficient was indicated between implicit shyness and implicit self-esteem $(r=-.72, p<.01)$. These results confirmed the testretest reliability and the criterion-related validity of the Japanese version of the shyness IAT, which is indicative of the validity of the test for assessing implicit shyness.

key words: implicit shyness, implicit association test, reliability, validity
\end{abstract}

\section{Introduction}

It is commonly believed that careful deliberation and conscious determination play an important role in regulating human thought and behavior. However, recent research is rebutting this assumption (e.g., [2], [3]). Bargh [4] proposed the auto-motive model, which posits that perceptions, cognitions, evaluations, emotions, objectives, and motives arise automatically. This theory has had such a huge impact on developments in the field of social psychology that it is sometimes referred to as the automaticity revolution [5].

Recently, a growing body of studies has been reported based on the implicit association test (IAT, [1]) to measure implicit self-concepts and attitudes (for a review, see [6]). Originally IATs were used to understand self-esteem [7] and stereotypes [8]. In recent research, however, this test has also been used to tap various personality traits, such as shyness ([9], [10]) and anxiety ([11], [12]), as well as the implicit theory of intelligence [13].

The IAT measures the degree to which a certain con-

\footnotetext{
Manuscript received December 7, 2012.

Manuscript revised April 7, 2013.

${ }^{\dagger}$ The author is with Sungshin Women's University, 249-1, Dongseon-dong 3-ga, Seongbuk-gu, Seoul, 136-742 Korea.

${ }^{\dagger}$ The authors are with Center for Research on Educational Testing (CRET), Tokyo, 163-1412 Japan.

${ }^{\dagger \dagger}$ The author is with The University of Tokyo, Tokyo, 113-0033

TH† The author is with University of Tsukuba, Tsukuba-shi, 305-

a) E-mail: va.ou.tu.peux@gmail.com

DOI: 10.1587/transfun.E96.A.1768
} Japan. 8577 Japan. cept is strongly associated with another concept, by recording the participants' response latency. The test typically consists of seven blocks, and in each block a respondent conducts a different task. The typical IAT involves two pairs of contrasting categories, such as self and others, and pleasant and unpleasant. In this task, for example, respondents indicate which of two categories a stimulus word belongs to by pressing one of two keys assigned to each category. When a stimulus word such as $I$ or $m y$ appears on the screen, for instance, the respondents choose the self category by pressing the key assigned to the self category, whereas when a stimulus word such as they or their appears on the screen, the respondents choose the others category by pressing the key assigned to that category. The stimulus words are presented in succession. This task is performed for target concepts such as self and others, as well as for attribute concepts such as pleasant and unpleasant. After finishing the task involving target concepts, the respondents conduct another task involving attribute concepts with stimulus words for pleasantness (e.g., happy, merry, good), or unpleasantness (e.g., unhappy, gloomy, bad). After performing these single-pair tasks, respondents conduct a dual-pair task in which each target category is paired with one of the two attribute categories (e.g., self + pleasant and others + unpleasant). When the stimulus word belongs to, for instance, either self or pleasant category, the key associated with self + pleasant is pressed. Then, a reversed task involving reverse pairings (self + unpleasant and others + pleasant) is also conducted. The averages for the two types of response latencies in the pairing tasks are measured and compared, formulating the comparative strength of conceptual association. For instance, implicit self-esteem is represented as the difference in average response latencies between the self + pleasant (others + unpleasant) task and the self + unpleasant (others + pleasant) task. A respondent's faster response to the former task (and slower response to the latter task) indicates a stronger implicit association between self and pleasantness, or the respondent's implicit self-esteem.

In this study, shyness is defined as "an affectivebehavioral syndrome characterized by social anxiety and interpersonal inhibition that results from the prospect or presence of interpersonal evaluation" [14]. Asendorpf et al. [10] assessed participants' shyness at an implicit, or an unconscious level with the IAT, as well as at an explicit, or a conscious level using a self-report scale and showed that shyness as measured by the two methods predicted different targets; implicit shyness measured by the IAT predicted uncon- 
trollable shy behaviors in interactional situations (e.g., tense postures and the frequency of touching the body), whereas explicit shyness measured by the self-report scale predicted controllable shy behaviors (e.g., not talking). Based on these results, Asendorpf et al. [10] proposed a double dissociation model in which each type of shyness measured by implicit and explicit methods predicted distinct targets. After confirming the predictive validity of the IAT, they concluded that the shyness IAT is useful for tapping individual differences in shyness.

Following Asendorpf et al.'s [10] paradigm, Aikawa and Fujii [9] also demonstrated the validity of the shyness IAT in a Japanese sample. The authors developed a Japanese version of shyness IAT for measuring implicit shyness among Japanese people. In addition to implicit shyness, explicit shyness of Japanese participants was rated by their peers, so that it could be measured more objectively. The results showed that the two types of shyness separately predicted participants' target behaviors, such as interpersonal tension and praise-seeking behavior. Building a structural equation model, the authors showed that implicit shyness predicted interpersonal tension (i.e., uncontrollable shy behavior), whereas explicit shyness predicted praise-seeking behavior (i.e., controllable shy behavior). In line with Asendorpf et al. [10], Aikawa and Fujii [9] replicated the double dissociation model on the predictability of the two types of shyness, though the authors cautioned that the conclusions of the study were limited by a statistically insufficient sample size $(N=28)$. To overcome this limitation, Fujii, Tanaka, Noguchi, and Aikawa [15] replicated the model and provided more robust evidence for the usefulness of the shyness IAT. Hence, it is fair to argue that the predictive validity of the shyness IAT for Japanese samples is well established.

Shyness plays an inhibitive role in interpersonal situations. For instance, those with high shyness often feel anxiety and cannot behave as they hope to in most interpersonal situations [16]. Furthermore, they rarely strike up a conversation with strangers, and even when they do so, they make little eye contact and get nervous [17]. Traditionally, shyness in these contexts has been measured by self-report scales; however, as Asendorpf et al. [10] showed, there is also a negative influence of implicit shyness, which is measured by the IAT, on interpersonal behavior (i.e., uncontrollable shy behavior). Taking the effect of implicit shyness into consideration, thus, it is important to investigate the validity of the shyness IAT in advancing research on shyness.

In the standard IAT, it will take merely several minutes for respondents to finish the test. When combining more than one IAT or adding self-report scales, however, it will take more time and is more likely to exhaust respondents accordingly. In addition to this disadvantage, experimenters need to prepare at least one computer for the test administration.

However, it is worth noting that the IAT has at least one advantage over self-report questionnaires in that the implicit measure is impervious to the effects of social desirability:
When respondents answer self-report questionnaires, they tend to present themselves as socially desirable, such as projecting themselves as being capable. In Aikawa and Fujii's [9] study and Fujii et al.'s [15] study, the results consistently showed that social desirability was associated with explicit shyness measured by self-report scales, but this was not the case with implicit shyness, as measured by the IAT.

In spite of this advantage however, important features of the test, such as its test-retest reliability and criterionrelated validity have remained untested. Firstly, the testretest reliability indicates the degree to which a test score is stable over time. It is usually calculated as the correlation of test scores between two points in time. Greenwald and Farnham [8] calculated the test-retest reliability correlation coefficient as being .52 after participants responded to the self-esteem IAT twice over an interval of eight-days. Reviewing 20 papers using the IAT, Lane et al. [7] reported that the test-retest reliability of the IAT ranged between .25 and .69, with .50 as the median. Secondly, the criterionrelated validity of a test indicates the degree to which the test score is related to other external criteria. This is usually judged by correlating the test score with a criterion test score. Glashouwer, de Jong, and Penninx [18] had participants respond to IATs for measuring anxiety and depression in a single session and reported a moderate positive correlation of .48 between the two tests. In another study, Schnabel, Banse, and Asendorpf [19] reported a positive correlation of .32 between anxiety and anger measured by the IAT.

This study was designed to confirm the test-retest reliability and criterion-related validity of the shyness IAT in a Japanese sample. For the test-retest reliability, the IAT was administered to Japanese participants twice with a one-week interval and the correlation between the two points of time was calculated to see if it remained nearly identical for all the participants over that period. As the criterion-related validity, we predicted a negative correlation between implicit shyness and implicit self-esteem (criterion), because previous research has consistently shown a negative association between the two traits as measured by explicit tests (e.g., [20], [21]). Self-esteem is defined as a positive affect towards the self and the sense of self-worth [22], which are contrary to shyness. On the one hand, those with high shyness are generally low in social skills and seem "awkward when they are smiling," "highly tense," and "not pleased" in interactional situations [18]. On the other hand, those with high self-esteem are generally high in social skills [23] and tend to show prosocial behavior [24]. Therefore, we expected that the negative correlation between the two traits, often demonstrated at the explicit level, would also be observed at an implicit level.

In order to deal with the problem of attenuation, we examined this correlation by estimating two latent variables of implicit shyness and implicit self-esteem, such that the correlation would not be weakened by measurement errors. 
Table 1 Procedure of Shyness-IAT.

\section{Method}

\subsection{Participants}

Adults $(N=59: 37$ men and 22 women; mean age, 21.83 years; $S D=2.52$ ) voluntarily participated in the study.

\subsection{Materials}

The following tests and scales were used.

Japanese version of shyness-IAT This study adopted a Japanese version of the shyness-IAT developed by [9], based on the creation by [10]. The procedure of the test is presented in Table 1. The current test was different from [9] in that Block 5 consisted of 20 trials in accordance with [25], instead of 40 trials as in [9]. We made this change to make the test parallel to another IAT for measuring selfesteem, as well as to lighten the burden on the participants. The IAT was administered on a computer. Identical stimuli as [9] were used in this study, as listed in Table 2. The test consisted of seven blocks. In the course of the IAT, paired categories (e.g., "Self" and "Others") were displayed constantly. Participants were asked to classify the target words that popped up in succession at the center of the screen into either the left or the right category by pressing the left or the right button.

In Block 1, participants were requested to classify the stimuli either as "Self" or "Others" target category, by pressing an assigned key (D key for a left category, $\mathrm{K}$ for a right category). In Block 2 participants were requested to classify the stimuli either as "Shy" or "Sociable" attribute category. In Blocks 3 and 4 participants were requested to respond to a combined task between Blocks 1 and 2; "Self" and "Shy" categories shared the left button response, whereas "Others" and "Sociable" shared the right button response. Block 3 served as a practice session for Block 4 . In Block 5 the position of "Self" and "Others" was reversed. In Blocks 6 and 7 the combination of Blocks 3 and 4 was reversed; "Others" and "Shy" categories shared a left button response, whereas "Self" and "Sociable" categories shared the right button response. In the standard IAT paradigm, Blocks 3 and 6 are termed as practice for Blocks 4 and 7 respectively; nevertheless, participants were not informed of the practice nature. In Blocks 1, 2, and 5, each stimulus of the respective categories were used two times. And they were presented randomly. In Blocks 3 and 6, each stimulus of target categories (i.e., "Self" or "Others") and those of attribute categories (i.e., "Shy" or "Sociable") were used one time and alternately presented. Indeed, in Blocks 4 and 7, all stimuli used two times and randomly presented; in each trial, the target word of the respective category was randomly selected.

In this shyness-IAT, shorter response latencies for Blocks 3 and 4, as compared to Blocks 6 and 7, were regarded as indicating a strong association between self and shyness, or implicit shyness. In order to control for the possible order effect, following previous literature (e.g., [25]),

\begin{tabular}{|c|c|c|c|c|}
\hline Block & Task & $\begin{array}{c}\text { Left } \\
\text { category }\end{array}$ & $\begin{array}{c}\text { Right } \\
\text { category }\end{array}$ & Trials \\
\hline 1 & $\begin{array}{c}\text { Target } \\
\text { classification }\end{array}$ & Self & Others & 20 \\
\hline 2 & $\begin{array}{c}\text { Attribute } \\
\text { classification }\end{array}$ & Shy & Sociable & 20 \\
\hline 3 & $\begin{array}{l}\text { Initial combined } \\
\text { task }\end{array}$ & $\begin{array}{c}\text { Self or } \\
\text { Shy }\end{array}$ & $\begin{array}{l}\text { Others or } \\
\text { Sociable }\end{array}$ & 20 \\
\hline 4 & $\begin{array}{c}\text { Initial combined } \\
\text { task }\end{array}$ & $\begin{array}{c}\text { Self or } \\
\text { Shy }\end{array}$ & $\begin{array}{l}\text { Others or } \\
\text { Sociable }\end{array}$ & 40 \\
\hline 5 & $\begin{array}{l}\text { Reversed target } \\
\text { classification }\end{array}$ & Others & Self & 20 \\
\hline 6 & $\begin{array}{c}\text { Reversed } \\
\text { combined task }\end{array}$ & $\begin{array}{c}\text { Others or } \\
\text { Shy }\end{array}$ & $\begin{array}{c}\text { Self or } \\
\text { Sociable }\end{array}$ & 20 \\
\hline 7 & $\begin{array}{c}\text { Reversed } \\
\text { combined task }\end{array}$ & $\begin{array}{c}\text { Others or } \\
\text { Shy }\end{array}$ & $\begin{array}{c}\text { Self or } \\
\text { Sociable } \\
\end{array}$ & 40 \\
\hline
\end{tabular}

Table 2 Category and Stimulus Words of Shyness-IAT

\begin{tabular}{|c|c|c|c|c|}
\hline Category & Self & Others & Shy & Sociable \\
\hline \multirow{5}{*}{ Stimulus } & Jibun & Tanin & $\begin{array}{l}\text { Uchiki na } \\
\text { (Bashful) }\end{array}$ & $\begin{array}{c}\text { Jishin no aru } \\
\text { (Assured) }\end{array}$ \\
\hline & Jishin & Shiriai & $\begin{array}{l}\text { Hikaeme na } \\
\text { (Moderate) }\end{array}$ & $\begin{array}{r}\text { Susunde suru } \\
\text { (Voluntary) }\end{array}$ \\
\hline & Onore & Chijin & $\begin{array}{c}\text { Mukuchi na } \\
\text { (Quiet) }\end{array}$ & $\begin{array}{c}\text { Daitan na } \\
\text { (Adventurous) }\end{array}$ \\
\hline & Watashi & Tomodachi & $\begin{array}{l}\text { Tamerai } \\
\text { gachi no } \\
\text { (Hesitant) }\end{array}$ & $\begin{array}{l}\text { Enryo no nai } \\
\text { (Uninhibited) }\end{array}$ \\
\hline & Wareware & Yuujin & $\begin{array}{l}\text { Enryo gachi } \\
\text { na (Reticent) }\end{array}$ & $\begin{array}{r}\text { Uchitoketa } \\
\text { (Familiar) }\end{array}$ \\
\hline
\end{tabular}

Note. The stimuli of "Self" and "Others" are expressed as Japanese pronunciations because their subtle nuances cannot be clearly manifested in English; all of these Japanese words indicate I/me, we/us, ,others, acquaintance, and friend.

we conducted the test in the above described order with half the participants, and in a different order with the other half by first presenting Blocks 5, 2, 6, and 7, followed by Blocks 1,3 , and 4 . For that purpose, we randomly assigned participants to each of the two tests.

Japanese version of self-esteem-IAT The Japanese version of self-esteem-IAT used by [26] was used with some modifications to the stimuli. Identical to the shynessIAT, the self-esteem-IAT consisted of seven blocks. In combined tasks (Blocks 3 and 4) stimuli were to be classified into either "Self or Comfortable" or "Others or Uncomfortable" superordinate category. Sample stimuli were "I," "friends," "incompetent," and "beautiful."

Trait Shyness Scale The 16-item Trait Shyness Scale (TSS) created and validated by [20] to measure trait shyness was used. Aikawa [20] has defined trait shyness as a group of syndromes characterized by an affective state of social anxiety and interpersonal inhibition that persists in an individual across many social situations. The scale consists of items such as, "I am not willing to make friends." This scale was responded by using a 5-point Likert scale 
anchored by extremely uncharacteristic of me (1) and extremely characteristic of me (5).

Rosenberg's Self-Esteem Scale We used the 10item Rosenberg's [27] Self-Esteem Scale (RSES) translated into Japanese by bilingual researchers according to Brislin's [28] committee approach. A sample item is, "I feel that I have a number of good qualities." This scale was responded on a 5-point Likert scale in the same manner as TSS.

\section{Procedure}

Participation in the study was voluntary. The experiment was conducted in a room equipped with computers. Before beginning the task, all participants were told that their participation was voluntary and that all personal information would be treated confidentially. Then participants gave their written informed consent to participate in the study. After giving back the consent form, no participant dropped out of the study. Then, participants were administered all the above tests and scales as one computerized package, which had been constructed using the experimental software Inquisit 3.0. Participants first conducted the two IATs, and then the two self-report scales. Considering possible order effects, the presenting order (shyness vs. self-esteem) within each measurement dimension was randomized. All participants completed the first wave of testing (Time 1), and did the same task one week later (Time 2). After finishing the task participants were fully debriefed and thanked.

\section{Results}

We used SPSS 17.0 and Amos 18.0 for the statistical analyses.

\subsection{Preliminary Analyses}

We averaged the 16 TSS item scores to obtain one index for explicit shyness. We also averaged the 10 RSES item scores for explicit self-esteem. Higher scores indicated a greater degree of explicit shyness and self-esteem. For implicit shyness and self-esteem, we calculated $D$ scores for the shyness-IAT and self-esteem-IAT, as proposed by Greenwald et al. [25]. Higher scores indicated a greater degree of implicit shyness and self-esteem. The $D$ scores were calculated according to the equation below.

$$
\begin{aligned}
D= & {\left[\left(\mathrm{MRL}_{\mathrm{B} 7}-\mathrm{MRL}_{\mathrm{B} 4}\right) / \mathrm{SD}_{\mathrm{B} 7, \mathrm{~B} 4}\right.} \\
+ & \left(\mathrm{MRL}_{\mathrm{B} 6}-\mathrm{MRL}_{\mathrm{B} 3} / \mathrm{SD}_{\mathrm{B} 6, \mathrm{~B} 3}\right] / 2
\end{aligned}
$$

where MRL, SD, B are mean response latency, standard deviation, and block, respectively. We calculated $D$ scores for implicit shyness using the following procedure. Data in Blocks 3, 4, 6, and 7 were used to obtain $D$ scores.

Following the proposal by Greenwald, et al. [25], we first excluded trials with responses of over 10000 milliseconds and then excluded participants that responded in less than 300 milliseconds for over $10 \%$ of the trials. No participants were excluded through this procedure. We imposed a penalty for error responses: In each block, response latencies for misjudged trials were replaced with mean response latency for only correct responses in the respective block plus 600 milliseconds. This replacement scheme was based on Greenwald et al.'s [25] findings. They empirically tested the way of dealing with error trials and concluded that adding 600 milliseconds, among others ranging from 200 to 1000 milliseconds, was the most desirable scheme in terms of lowering a correlation between $D$ score and overall response time; the correlation is not desirable because those with slower response time would produce higher $D$ score accordingly. Then, we computed the pooled standard deviation for each participant for all the trials in Blocks 3 and 6, as well as in Blocks 4 and 7. After that, the mean latencies for all the responses in each of the Blocks 3, 4, 6, and 7 were computed using the means, some of which were replaced due to error penalty. Differences in mean latency were computed by comparing between Blocks 3 and 6 and between Blocks 4 and 7, in such a way that the mean latency for Blocks 3 and 4 (in which participants conducted the "self + shy" or "others + sociable" combined task) was subtracted from that for Blocks 6 and 7 (in which participants conducted the reverse combined task). For participants that did the test in the reverse order, the mean latency for Blocks 6 and 7 (in which the participants conducted the "self + shy" or "others + sociable" combined task) was subtracted from that for Blocks 3 and 4 (in which the participants conducted the reverse combined task). Lastly, we divided each mean score by its associated pooled standard deviation for each participant. $D$ scores were obtained by averaging the two resulting scores. The same formula was applied for calculating $D$ scores for implicit self-esteem. Those with higher implicit shyness, or implicit self-esteem showed higher $D$ scores.

4.2 Correlation Coefficients between Indicators and TestRetest Reliability of Shyness-IAT

A significant positive correlation $(r=.67, p<.01)$ was found between implicit shyness at Time 1 and that at Time 2.

Descriptive statistics of all the indicators are presented in Table 3 and correlation coefficients between indicators are presented in Table 4. As can be seen in Table 3, TSS and RSES revealed high internal consistency. And as can be seen in Table 4, there was a significant negative correlation between TSS and RSES, as well as between shyness-IAT and self-esteem-IAT at both points of time.

4.3 Correlation between Implicit Shyness and Implicit Self-Esteem

Attenuation is an occasionally encountered statistical problem, in which correlations are weakened by measurement errors. To overcome this problem, we postulated two latent variables, implicit shyness and implicit self-esteem respectively, estimated by $D$ scores at two points of time. We cre- 
Table 3 Descriptive statistics of indicators.

\begin{tabular}{lccc}
\hline & $M$ & $S D$ & $\alpha$ \\
\hline${\text { Shyness-IAT }(D \text { score })^{1}}^{1}$ & -0.06 & 0.48 & - \\
Shyness-IAT (Latency) $^{1}$ & 790.98 & 175.03 & - \\
Self-esteem-IAT $\left(D\right.$ score) $^{1}$ & 0.58 & 0.53 & - \\
Self-esteem-IAT(Latency) $^{1}$ & 749.20 & 176.37 & - \\
${\text { Shyness-IAT }(D \text { score })^{2}}^{2}$ & -0.22 & 0.39 & - \\
Shyness-IAT (Latency) $^{2}$ & 758.75 & 240.86 & - \\
${\text { Self-esteem-IAT }(D \text { score })^{2}}^{2}$ & 0.53 & 0.47 & - \\
Self-esteem-IAT (Latency) $^{2}$ & 665.76 & 127.29 & - \\
TSS $^{1}$ & 3.09 & 0.64 & .89 \\
RSES $^{1}$ & 3.41 & 0.59 & .87 \\
TSS $^{2}$ & 3.05 & 0.61 & .90 \\
RSES $^{2}$ & 3.40 & 0.56 & .88 \\
\hline
\end{tabular}

Note: IAT $=$ Implicit Association Test. Superscripts indicate the time of administration.

Table 4 Inter-correlations between indicators.

\begin{tabular}{lccccccc}
\hline & 2 & 3 & 4 & 5 & 6 & 7 & 8 \\
\hline 1 Shyness-IAT & $-.53^{* *}$ & $.43^{* *}$ & $-.47^{* *}$ & .03 & .12 & .02 & .10 \\
2 Self-esteem-IAT & - & $-.31^{*}$ & $.67^{* *}$ & -.03 & -.03 & .05 & .00 \\
3 Shyness-IAT & & - & $-.30^{*}$ & .10 & .02 & .06 & .01 \\
4 Self-esteem-IAT & & & - & -.13 & -.01 & -.08 & .00 \\
5 TSS $^{1}$ & & & & - & $-.36^{* *}$ & $.87^{* *}$ & $-.40^{* *}$ \\
6 RSES $^{1}$ & & & & & - & $-.37^{* *}$ & $.92^{* *}$ \\
7 TSS $^{2}$ & & & & & - & $-.40^{* *}$ \\
8 RSES $^{2}$ & \\
\hline Note: IAT $=$ Implicit Association Test. Superscripts indicate the time of & \\
administration. ${ }^{* *} p<.01,{ }^{*} p<.05$.
\end{tabular}

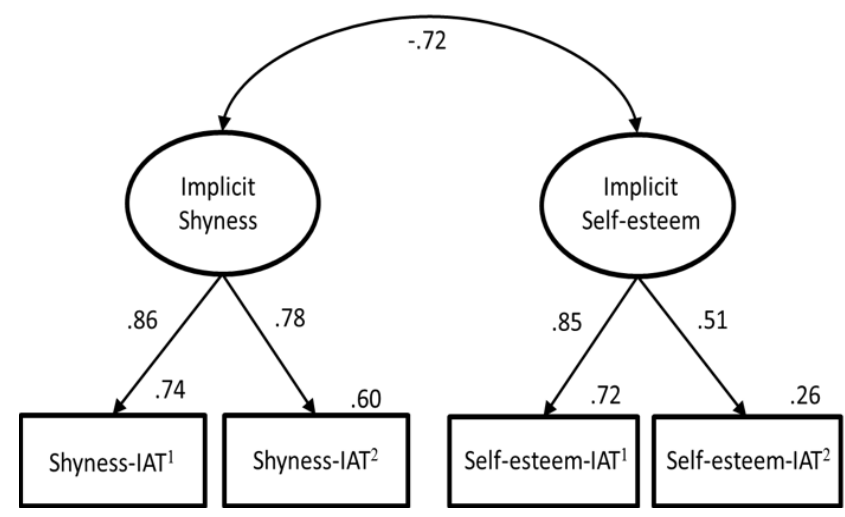

Fig. 1 Structural equation model

Note $:$ IAT = Implicit Association Test. Superscripts indicate the time of administration.

ated a structural equation model as illustrated in Fig. 1, with the following fit indices: $\chi^{2}=.064(p=.801), \mathrm{GFI}=.999$, AGFI $=.995, \mathrm{CFI}=1.000$, RMSEA $=.000$. These indices indicated that the model fitted well with our data. As can be seen in this figure, the two latent variables (i.e., implicit shyness and implicit self-esteem) estimated by $D$ scores had a strong negative correlation with each other $(r=-.72, p<$ $.01)$.

\section{Discussion}

This study was designed to establish the test-retest reliability and criterion-related validity of the Japanese version of the shyness-IAT. The results indicated a test-retest reliability of .67, which is lower than that of conventional self-report scales. However, it could be considered sufficiently high within the realm of implicit measures, median of which is reported as .50 as Lane et al.'s [7] meta-analysis. Therefore, it is considered that the Japanese shyness-IAT could identify implicit shyness with sufficient stability, independently of when the test was administered.

The results also showed that, based on a structural equation modeling for eluding the issue of attenuation, the criterion-related validity of shyness-IAT was -.72 , as calculated by its correlation with the criterion of implicit selfesteem. Although the criterion-ralated validity for shyness should not be limited to self-esteem, previous literature has consistently shown a negative relationship between shyness and self-esteem using self-report scales (e.g., [20], [21]). Moreover, in this study, we found a negative relationship between shyness and self-esteem at an implicit level.

The Japanese shyness-IAT was demonstrated to be valid and reliable, with sufficient test-retest reliability, criterion-related validity, and predictive validity ([9], [15]). Moreover, past studies have confirmed the imperviousness of the test to the effects of social desirability ([9], [15]). Therefore, it is concluded that the shyness-IAT is an appropriate indicator of implicit shyness.

Besides IAT, there already exist other implicit measures such as thematic apperception test, stroop test, and evaluative priming. These tests, however, have been shown to hold the following practical problems: requiring respondents' repeated practices and low test-retest reliability. Thus, as an alternative to these tests, IAT has received researchers' attention because of its easy scoring and high test-retest reliability.

This study validated the Japanese version of the shyness-IAT, which was originally translated from English into Japanese by [9]. As to the level of shyness and its closely related concept, self-esteem, its cultural differences have been suggested. In case of self-esteem, the level has consistently been reported higher in Western culture than in East Asian cultures (e.g., [29], [30]). Also, as Zimbardo [31] argued, the level of shyness among East Asian people, especially Japanese, is most likely to be reported higher, as compared with other cultures. As to impression on peers, there can be interesting cultural differences. In Western culture, expressing one's own high shyness is socially undesirable [10], whereas doing that does not always lead to that negative impression among Japanese [32]. Rather, Sawaumi, Fujii, \& Aikawa [32] implied that those with high shyness can be regarded as personable by Japanese.

From a cross-cultural perspective, also, there might be cultural differences in subtle nuances implied by used words in each language. We cannot say for sure that stimuli used in 
[9] and those used in [10] have equivalent nuances. Thus, as a caveat, this study validated the use of shyness-IAT merely for Japanese samples. Conversely, it is also fruitful to extend the use of the IAT to other cultural contexts such as China and South Korea, which would enable examining cultural differences within East Asian cultures.

Studies on implicit self-concepts and attitudes have a history of only 20 years [33], which is very short compared to conventional self-report questionnaires. As a result, there are many variables of implicit tests that remain unknown. It is suggested that future research should be conducted not only about shyness, but also on other self-concepts and attitudes using valid and reliable implicit measures such as IATs. This is expected to result in a new understanding of implicit, or unconscious processes.

\section{Acknowledgments}

This study was supported by Center for Research on Educational Testing (CRET).

\section{References}

[1] A.G. Greenwald, D.E. McGhee, and J.K. Schwartz, "Measuring individual differences in implicit cognition: The implicit association test," J. Personality and Social Psychology, vol.74, no.6, pp.14641480, 1998.

[2] M. Oikawa and H. Oikawa, "Unconscious thinking, doing, and wanting: A review of mechanisms and moderators of assimilation and contrast effects," Japanese Psychological Review, vol.53, no.4, pp.483-496, 2010. (in Japanase)

[3] S. Simojo, Subliminal Impact, Chikuma Shobo, 2008. (in Japanase)

[4] J.A. Bargh, "Auto-motives: Preconscious determinants of social interaction," in Handbook of Motivation and Cognition: Foundations of Social Behavior, vol.2, pp.93-130, Guilford Press, New York, NY, US, 1990.

[5] T. Ikegami, "Automatic processing and control processing," in Psychology of Social Cognition, ed. M. Karasawa, T. Ikegami, K. Karasawa, and H. Ohira, pp.130-151, Nakanishiya Shuppan, Kyoto, Japan, 2001. (in Japanase)

[6] K.A. Lane, M.R. Banaji, B.A. Nosek, and A.G. Greenwald, "Understanding and using the Implicit Association Test: IV: What we know (so far) about the method," in Implicit Measures of Attitudes, ed. B. Wittenbrink and N. Schwarz, pp.59-102, Guilford Press, New York, NY, US, 2007.

[7] A.G. Greenwald and S.D. Farnham, "Using the implicit association test to measure self-esteem and self-concept," J. Personality and Social Psychology, vol.79, no.6, pp.1022-1038, 2000.

[8] L.A. Rudman, A.G. Greenwald, and D.E. McGhee, "Implicit selfconcept and evaluative implicit gender stereotypes: Self and ingroup share desirable traits," Personality and Social Psychology Bulletin, vol.27, no.9, pp.1164-1178, 2001.

[9] A. Aikawa and T. Fujii, "Using Implicit Association Test (IAT) to measure implicit shyness," Japanese J. Psychology, vol.82, no.1, 4148, 2011. (in Japanase)

[10] J.B. Asendorpf, R. Banse, and D. Mücke, "Double dissociation between implicit and explicit personality self-concept: The case of shy behavior,' J. Personality and Social Psychology, vol.83, no.2, pp.380-393, 2002.

[11] B. Egloff and S.C. Schmukle, "Predictive validity of an implicit association test for assessing anxiety," J. Personality and Social Psychology, vol.83, no.6, pp.1441-1455, 2002.

[12] T. Fujii, "Predictive validity of the IAT-anxiety," Japanese J. Person- ality, vol.20, no.1, pp.57-60, 2011. (in Japanase)

[13] T. Fujii and H. Uebuchi, "Assessment of participants' theories of intelligence: Reliability and validity of the Implicit Association Test," Japanese J. Educational Psychology, vol.58, no.3, pp.264275, 2010. (in Japanase)

[14] M.R. Leary, "Affective and behavioral components of shyness: Implications for theory, measurement, and research," in Shyness: Perspectives on research and treatment, ed. W.H. Jones, J.M. Cheek, \& S.R. Briggs, pp.27-38, Plenum Press, New York, NY, 1986.

[15] T. Fujii, C. Tanaka, Y. Noguchi, and A. Aikawa, "An attempt to measure implicit and explicit shyness," Poster presented at the 27th International Congress of Applied Psychology, Melbourne, Australia July 2010.

[16] J.B. Asendorpf, "Videotape reconstruction of emotions and cognitions related to shyness,' J. Personality and Social Psychology, vol.53, no.3, pp.542-549, 1987.

[17] J.M. Cheek and A.H. Buss, "Shyness and sociability," J. Personality and Social Psychology, vol.41, no.2, pp.330-339, 1981.

[18] K.A. Glashouwer, P.J. de Jong, and B.W. J.H. Penninx, "Predictive validity of automatic self-associations for the onset of anxiety disorders," J. Abnormal Psychology, vol.120, no.3, pp.607-616, 2011.

[19] K. Schnabel, R. Banse, and J.B. Asendorpf, "Assessment of implicit personality self-concept using the implicit association test (IAT): Concurrent assessment of anxiousness and angriness," British J. Social Psychology, vol.45, no.2, pp.373-396, 2006.

[20] A. Aikawa, "A study on the reliability and validity of a scale to measure shyness as a trait," Japanese J. Psychology, vol.62, no.3, pp.149-155, 1991. (in Japanase)

[21] M. Goto, "Social psychological studies for shyness," Japanese J. Interpersonal and Social Psychology, vol.1, 81-91, 2001.

[22] T. Ito, "Self-esteem and self-evaluation," in Social psychology of ego and self, ed. M. Funatsu and K. Ando, pp.96-111, Hokuju Shuppan, Tokyo, 2002. (in Japanase)

[23] R.E. Riggio, B. Throckmorton, and S. DePaola, "Social skills and self-esteem," Personality And Individual Differences, vol.11, no.8, pp.799-804, 1990.

[24] C. Batson, M.H. Bolen, J.A. Cross, and H.E. Neuringer-Benefiel, "Where is the altruism in the altruistic personality?," J. Personality and Social Psychology, vol.50, no.1, pp.212-220, 1986.

[25] A.G. Greenwald, B.A. Nosek, and M.R. Banaji, "Understanding and using the implicit association test: I. An improved scoring algorithm," J. Personality and Social Psychology, vol.85, no.3, pp.197216, 2003.

[26] T. Sawaumi, Y. Sugiyama, S. Yamaguchi, Y. Xu, and H. Cai, "The relationship between explicit/implicit self-esteem and other individual traits (2)," Poster presented at the 49th Annual Meeting of Japanese Society of Social Psychology, pp.192-193, Nov. 2008 (in Japanase).

[27] M. Rosenberg, Society and the adolescent self-image, Princeton University Press, Princeton, NJ, 1965.

[28] R.W. Brislin, "Translation and content analysis of oral and written materials," in Handbook of cross-cultural psychology, ed. H.C. Triandis and J.W. Berry, vol.2, Methodology, pp.389-444, Allyn and Bacon, Boston, 1980.

[29] S.J. Heine, D.R. Lehman, H.R. Markus, and S. Kitayama, "Is there a universal need for positive self-regard?," Psychological Review, vol.106, no.4, pp.766-794, 1999.

[30] S. Yamaguchi, A.G. Greenwald, M.R. Banaji, F. Murakami, D Chen, K. Shiomura, C. Kobayashi, H. Cai, and A. Krendl, "Apparent universality of positive implicit self-esteem," Psychological Science, vol.18, no.6, pp.498-500, 2007.

[31] P.G. Zimbardo, Shyness: What it is, what to do about it, AddisonWesley, Massachusetts, 1977.

[32] T. Sawaumi, T. Fujii, and A. Aikawa, "An experimental study on interpersonal impressions of shy people: Focus on positive aspects of shy people," Poster presented at the 76th Annual Meeting of Japanese Psychological Association, p.34. (in Japanase) 
[33] Y. Ozaki, "Formation and change of implicit attitude through classical conditioning: A review of empirical evidence," Behavioral Science Research, vol.49, no.1, pp.53-61, 2010. (in Japanase)

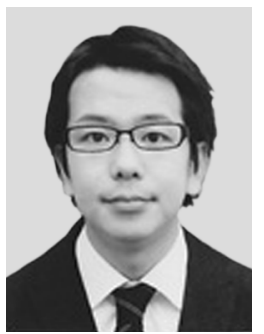

Tsutomu Fujii received the B.A. and M.Ed. degrees from Tokyo Gakugei University in 2007 and 2009, respectively. Between 2010-2012, He worked at Department of psychology, Faculty of letters, at Gakushuin University. He is now with Department of Japanese language and literature in Sungshin Women's University, Korea.

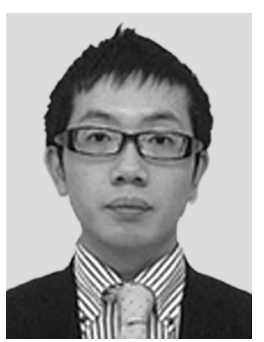

Takafumi Sawaumi received the B.A. and M.A. degrees in social psychology from the University of Tokyo in 2006 and 2008, respectively. He is currently in Department of Social Psychology, Graduate School of Humanities and Sociology, at The University of Tokyo, studying for his Ph.D. in social psychology.

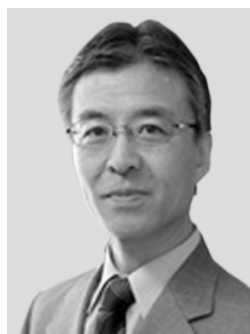

Atsushi Aikawa received the M.A. and a Doctor of Psychology degree from Hiroshima University, Japan, in 1980 and 1995, respectively. He is a Professor at Graduate School of Comprehensive Human Sciences at University of Tsukuba, Japan. His research interest is in the area of social skills and social skills training. 